\title{
El museo analizado desde la sociología simétrica
}

The museum analyzed from symmetric sociology

Le musée analysé à partir de la sociologie symétrique

\section{Scarlet Rocío Galindo Monteagudo}

\section{OpenEdition}

\section{Journals}

Edición electrónica

URL: http://journals.openedition.org/iss/2068

DOI: $10.4000 /$ iss.2068

ISSN: 2306-4161

\section{Editor}

ICOM - International Council of Museums

\section{Edición impresa}

Fecha de publicación: 1 agosto 2020

Paginación: 121-131

ISBN: 978-2-491997-11-3

ISSN: 2309-1290

\section{Referencia electrónica}

Scarlet Rocío Galindo Monteagudo, «El museo analizado desde la sociología simétrica », ICOFOM Study Series [En línea], 48-1 | 2020, Publicado el 01 agosto 2020, consultado el 08 agosto 2020. URL http://journals.openedition.org/iss/2068 ; DOI : https://doi.org/10.4000/iss.2068 


\section{El museo \\ analizado desde la sociología simétrica}

\section{Scarlet Rocío Galindo Monteagudo Museo Nacional de la Acuarela - Ciudad de México, México}

El presente ensayo responde las interrogantes: ¿Qué es la Teoría del Actor Red (TAR)?, ¿qué es la sociología simétrica?, ¿qué utilidad tiene el uso de esta teoría en la investigación museológica? En él se revisarán aplicaciones de la TAR como marco teórico en investigaciones museológicas y en la creación de exposiciones. Con la finalidad de aclarar algunos conceptos y revisar la utilidad del uso de esta teoría en la construcción del futuro de la museología.

Palabras clave: Teoría del actor red, sociología simétrica, experimentalista.

A B STRACT

The museum analyzed from symmetric sociology

The present essay answers the following questions: What is Actor Network Theory (ANT)? What is symmetric sociology? How useful could be this theory for museological research? It reviews the ANT's use as a theoretical framework in museological studies, as well as in 
the creation of exhibitions. Its purpose is to clarify some concepts and review its usefulness in the construction of museology future.

Keywords: Actor network theory, symmetric sociology, experimentalist.

\section{RÉSUMÉ}

\section{Le musée analysé à partir de la sociologie symétrique}

Le présent essai répond aux questions suivantes : Qu'est-ce que la théorie de l'acteur-réseau (ANT) ? Qu'est-ce que la sociologie symétrique ? Quelle est l'utilité de cette théorie dans la recherche en muséologie? Il revue l'utilisation de la théorie de l'acteur-réseau en tant que cadre théorique dans les études muséologiques, ainsi que dans la réalisation d'expositions. Il vise à clarifier certains concepts et revoir son utilité dans la construction du futur de la muséologie.

Mots-clés : théorie de l'acteur-réseau, sociologie symétrique, expérimentateur.

\section{丈}

\section{Introducción}

El presente texto se enfoca en como la Teoría del Actor Red ha sido utilizada en las investigaciones en museos en los últimos años, buscando colocarla como una teoría metodológica que puede apoyar a los estudios museológicos.

El común denominador de todas las investigaciones mencionadas en este texto es que analizan al museo desde lo microsocial ${ }^{\mathrm{r}}$, experimentando en este espacio a través de los actores humanos y no humanos las formas de representación, que un objeto puede dar múltiples interpretaciones y dependerá de los profesionales en museos los discursos que queramos que sean presentados y representados en él.

\footnotetext{
I. La sociedad tiende a explicarse desde arriba, es decir desde estructuras como son las masas y los sistemas a esto se le llama la macrosociología, algunos ejemplos de estudios sociales con este tipo de estructuras son los determinismos culturales de Parsons, o los sistemas de Luhmann. Contra ello se puede ver la sociedad explicada desde abajo, es decir, desde el grupo o individuo, a esto se le llama microsociología. Dentro de los estudios relacionados con este enfoque están el interaccionismo simbólico de Blumer y la etnometodología de Garfinkel. Es el estudio de la sociedad desde los vínculos sociales elementales, es decir, las estructuras, relaciones internas y formas de organización de pequeños grupos.
} 


\section{¿Qué es la Teoría del Actor Red (TAR)?}

A mediados de los años ochenta Bruno Latour, Michel Callon, John Law, Madeleine Akrich, Andy Barry, Annemarie Mol, Antoine Hennion, entre otros teóricos crearon la Teoría del Actor Red (TAR) (Latour, 2008). También conocida como la Teoría del Enrolamiento o Sociología de la Traducción, que tiene su origen en los estudios sociales de la ciencia de finales de r970 denominados el Programa Fuerte de la Ciencia (PFC), cuyo principal precursor fue David Bloor (1976). También está relacionada con la Escuela de Edimburgo que buscaba una nueva forma de analizar la ciencia en contraposición de las sociologías clásicas. Del Programa Fuerte de la Ciencia (PFC), de David Bloor (1942) y Barry Barnes (1943), surgirá la Nueva Sociología del Conocimiento Científico (NSCC) en la que se observa la flexibilidad interpretativa de los hechos, su precursor Barry Barnes decía que el conocimiento científico es "aquello que la gente considera como tal" (I995) y por ello para su estudio, David Bloor propuso en el texto: Knowledge and Social Imagery (1976) cuatro principios para una explicación científica de la naturaleza y el cambio del conocimiento científico que son: la causalidad, la imparcialidad, la simetría y la reflexividad, los cuales se explican a continuación:

- La causalidad. Se refiere a estar preocupado por las condiciones que dan lugar a la creencia o estados de conocimiento.

- La imparcialidad. Se refiere a explicar tanto los éxitos como los fracasos, lo racional como lo irracional; lo verdadero como lo falso; utilizando un mismo estilo de explicación, el cual se puede dar siguiendo un mismo número de pasos metodológicos.

- La simetría generalizada. Tiene como objetivo explicar los puntos de vista y argumentos enfrentados en una controversia científica, tecnológica, histórica o artística, a partir de los mismos términos, ya que los ingredientes de las controversias son una mezcla de consideraciones sobre la naturaleza y la sociedad y esto se logra utilizando el mismo repertorio (Callon, I986, p. 26I-262).

- La reflexividad. Bloor menciona que en principio sus patrones de explicación tendrían que ser aplicables a la propia sociología y en este caso a la museología, ya que se buscan explicaciones generales. Se cumple al lograr que el científico social no se avergüence de ver teorías y métodos que emanan de la sociedad como el producto de influencias, recursos colectivos y particularmente de la cultura y sus circunstancias actuales (Bloor, 1976 , p. I-38).

Para la TAR lo importante, no solo es mostrar el proceso de gestación de lo social. En sus casos de estudio además de darse a conocer las capacidades y trucos profesionales que ayudan a que lo social emerja, al ser simétrica también se debe mostrar aquello que en otras investigaciones fue editado o eliminado. De tal forma que podremos ver cómo las cosas pudieron darse de manera diferente, 
el por qué se tomaron esas decisiones y las fallas o errores presentados en el proceso, algo que no sucede con frecuencia en un producto de investigación terminado (Latour, 2008).

Los teóricos de la TAR han utilizado los principios del PFC para poder desligarse de lo que es considerado el "verdadero conocimiento" y la "falsa creencia", con la intención de observar lo social de manera diferenciada (Latour, r999, p. II4), abogan por un trabajo etnográfico ya que están influenciados por otro programa surgido en los años ochenta en la Universidad de Bath, el Programa Empírico del Relativismo (PEDR), impulsado por Harry Collins (1943) y Trevor Pich (1952), este programa se encuentra ubicado en el Constructivismo Social y demuestra la flexibilidad interpretativa de los resultados experimentales; así como los mecanismos sociales, retóricos e institucionales que la limitan y favorecen al cierre de controversias científicas, promoviendo el consenso de la verdad. También revisa los mecanismos de cierre de estas controversias que se relacionan con el medio sociocultural y político. Pero para comprender mejor lo anterior, definiré ¿qué son las controversias?

Según el teórico Michel Callon (1945) las controversias, son el origen de las convenciones sociales, "los consensos, convenciones o juicios [...], es decir, aquellos argumentos y puntos de vista contradictorios que llevan a los diferentes actores que comprenden una red a proponer diferentes versiones del mundo social y natural" (Callon, 1986).

La TAR, entonces analiza las redes ${ }^{1}$ en las que los actantes ó actores ${ }^{2}$ humanos y no humanos (máquinas, objetos, animales, textos e híbridos, entre otros) se asocian (Ritzer, 2005, p. I-2). Para el caso de los museos asociar a los objetos resulta un elemento de gran importancia, ya que la conservación del patrimonio se encuentra en ellos. La TAR utilizará del Programa Fuerte de la Ciencia sus principios para el análisis de casos desde la denominada sociología simétrica, para

\footnotetext{
I. A la palabra red en muchas ocasiones se le ha dado un significado apegado a lo tecnológico. Sin embargo, su uso proviene de la palabra réseau que utilizó Diderot para describir la materia y los cuerpos con el fin de evitar la división cartesiana entre materia y espíritu. Las redes no se encuentran en términos dimensionales sino como nodos, por lo que se debe pensar en las sociedades "con un carácter fibroso, filiforme, nervudo, viscoso capilar que nunca es capturado por las nociones de niveles, capas, territorios, esferas, categorías, estructuras o sistemas" (Bloor, 1976).

2. 'Un 'actor' es una definición semiótica, 'un actante' que es, algo que actúa o la actividad que se le concede por otros. No implica una especial motivación de actores humanos individuales, ni de los seres humanos en general. Un actante puede, literalmente, ser cualquier cosa con tal de que se le conceda ser la fuente de una acción" (Latour, 1996, p. 7) y cuando se habla de actores no humanos, no se trata de analizar a los objetos en sí mismos sino "...en las redes de las que forman parte. Objetos, entidades, actores, procesos 'todos son efectos semióticos: nodos de una red que no son más que conjuntos de relaciones; o conjuntos de relaciones de relaciones. Empújense la lógica un paso más allá: los materiales están constituidos interactivamente; fuera de sus interacciones no tienen existencia, no tienen realidad. Máquinas, gente, instituciones sociales, el mundo natural, lo divino' todo es un efecto o un producto" (Law, John \& Mol, Annemarie, 1994, p. 277).
} 
ella la revisión de los factores sociales no es suficiente para explicar la dinámica de la sociedad, por ello recurre a los medios heterogéneos, es decir, toma en consideración también a los actores no humanos, ya que las consecuencias de lo que se hace con estos actores se vuelven efectos de negociaciones, por lo que podemos decir que es una teoría reduccionista y relativista, cercana a los llamados estudios sociales de laboratorio (Núñez, 20II).

A continuación, se mencionarán algunas investigaciones que abordan el museo desde esta perspectiva.

\section{El uso de la TAR en las investigaciones museológicas}

Un texto clásico en el uso de la TAR es "From blindness to blindness: museums, heterogeneity and the subject" (1999) en él, Kevin Hertherington analiza el devenir de los objetos (actores no humanos) y sujetos (actores humanos) en el museo y el concepto de "heterogeneidad". Presenta la historia del ojo y la percepción, dividiéndola en cinco momentos:

En el primero, habla de la perspectiva lineal en el Renacimiento, relacionándola con el infinito. El espacio museal es entonces un lugar donde el sujeto está separado del mundo material, viendo desde una posición privilegiada y la heterogeneidad es representada en términos de similitud.

En el segundo, rompe con esta idea y el museo es visto como heteróclito, separa lo diferente. El cuadro de las Meninas de Velázquez es un ejemplo de ello, el sujeto no es presentado en términos de similitud y por ello los objetos tienen la necesidad de ser clasificados, a este momento pertenecen los gabinetes de curiosidades como templos de memoria.

Después de ello, el autor nos habla en un tercer momento de la modernidad, cuando el sujeto es un panóptico y el museo un lugar de vigilancia que controla la heterogeneidad del objeto comenzando a representar formas de alta cultura, ya que civiliza, un texto asociado a este momento es "The birth of the museum. History, theory and politics" de Tony Bennett (1995).

En un cuarto momento, relaciona al movimiento dadaísta y la TAR, al dar nuevamente heterogeneidad a los objetos en los museos por el lugar que ocupan, el autor menciona entonces "Lo que es heterogéneo dentro del sujeto fluye del ojo al mundo material de las cosas y se celebra como fuente de agencia" (Hetherington, I999, p. 7I).

Finalmente, nos habla del futuro, un quinto momento en los museos en el que propone que el sujeto salga de ver a los objetos con los ojos y lo haga desde el mundo de las ideas.

En el texto titulado: "Behind the Scenes at the Science Museum" (2002), Sharon Macdonald sigue a los actores de una exposición que ocurre en el Museo de Ciencias de Londres, y lo hace a través de una etnografía. Ella nos comenta que la exposición respondía a las preguntas ¿qué hay de ciencia en la comida?, 
¿cuáles son los objetos más poderosos para representarla?, ¿qué es único en la comida inglesa? y ¿qué encontrarán los visitantes de entretenido o interesante en la comida?

La investigación analiza el papel de cada participante o actor, tales como el staff y los patrocinadores; se revisa su influencia en la toma de decisiones. Destaca en ella como ante la falta de una idea general de lo que es el museo, se genera el "multi-museo" o los "museos dentro de la idea de museo" (2002, p. 59), conceptos que ayudaron a los curadores e investigadores de la muestra a aportar formas más flexibles de trabajar los temas presentados en sus salas, no teniendo un concepto de representación único y no relacionando este espacio con la idea hegemónica de ciencia.

El museo presenta a la ciencia en la cotidianidad, intentando democratizarla al utilizar objetos de una forma sencilla favoreciendo con ello una construcción diferente del concepto, más cercano al público, más didáctico y menos sacralizado. Una de las conclusiones a las que llega este texto es que la historia solo puede ser contada a partir de ejemplos poderosos (Macdonald, 2002). Sin embargo, aun cuando la autora en principio no toma en cuenta la dimensión política y de mercado que le daba el potenciar a las marcas presentadas, el revisar al museo detrás de escena le ayuda a visibilizar estas controversias y su importancia en el desarrollo de la muestra.

En 2005 , Richard Toon vincula la arquitectura de museos con el concepto de "caja negra", término que se refiere a una expresión que usan los cibernéticos cuando un artefacto es demasiado complejo y en lugar de explicarlo se dibuja una caja de la que únicamente se ven sus entradas y salidas. Entonces abre la "caja negra" de los espacios museales dedicados a la ciencia llegando a la conclusión de que existen dos tipos de museos o centros de ciencia: I) aquellos que dan una "rebanada de la historia" y particularmente de la comunidad y 2) aquellos que presentan reglas universales y principios que trascienden el tiempo y espacio, a estos últimos al carecer de elementos identitarios los relaciona con los "no lugares" de Marc Augé (Toon, 2005, p. 26-38). El texto es entonces un análisis de actores no humanos, revisando los edificios y discursos museográficos. El autor plantea que para que estos museos "no lugares" lleguen a ser 'espacios', deben hacer la distinción que Michael de Certeau menciona en su texto "Relatos del espacio" y que dice: "En suma, el espacio es un lugar practicado. De esta forma, la calle geométricamente definida por el urbanismo se transforma en espacio por intervención de los caminantes..." (Certeau, 2007), invitándonos a generar comunidad.

\footnotetext{
I. Según Augé "si un lugar puede definirse identidad, relacional e histórico, un espacio que no puede definirse como espacio de identidad ni como relacional ni como histórico, definirá un no lugar" (Augé, 1996, p. 83).
} 
Otros usos de esta teoría se pueden ver en los textos de Larry Kang "Object/ Shadow-Notes on a developing art form" (Kang, 2006) y el de Rasmus Kjærboe, "Collecting the modern" (2016).

En el primero, el artista Larry Kang desarrolla un mapeo de actores dentro del propio devenir de su obra. Para ello crea una nueva categoría para su escultura llamada object/shadow. En el texto reflexiona sobre su devenir como artista, ocurrido por su facilidad para dibujar; también nos habla de sus esculturas, el por qué de la utilización de ciertos materiales como el acero, el por qué a algunas de sus piezas les coloca marcos y el cómo las sombras forman parte de su obra. Para dicha exposición sugiere incluso en un principio poner una luz fluorescente para evitar las sombras, pero posteriormente las ocupa como parte de la obra forzándose entonces a concebir la dimensión espacial en donde se colocarían. También analiza la percepción de los visitantes de estas sombras, la cual en un principio es nula, son interpretadas como un dibujo en la pared, viendo como se sorprende el público al saber que aun cuando forman parte de la obra, no son un dibujo o parte del acero que la conforma, sino la iluminación y el espacio, todo esto mediante el ensayo y error de una muestra experimental, propuesta por el propio artista.

Mientras que Rasmus Kjærboe, desarrolla otro mapeo de asociaciones en relación con la adquisición de la colección modernista del museo de Ordrupgaard fundado en I9I8 y que contiene piezas de I9I7 a I923, el cual está ubicado en el municipio de Gentofte al norte de Copenhague.

Dividiendo su estudio en la exposición; análisis de sus visitantes; la curaduría; los coleccionistas; la forma en que los objetos son exhibidos; los museos que surgieron en ese momento histórico, con los que hace una comparación; entre otras temáticas. Enfatiza en las redes formadas entre coleccionistas, en como el Estado danés interviene al adquirir el museo, asi como su relación con el préstamo de piezas para exhibiciones modernistas en la actualidad, también toca el tema de la venta de obra de este movimiento, revisando finalmente en un epílogo que está haciendo el museo actualmente y lo que piensa podría ser su futuro. La conclusión a la que llega esta tesis es que coleccionar arte y realizar un museo privado depende de redes: gente, cosas, organizaciones y técnicas y que lo que aun hace a este espacio distinto ante otros es el edificio que lo contiene y la atmósfera que presenta.

En 2016, Laurie Waller escribe: "Curating actor-network theory: testing objectoriented sociology in the science museum", en la que investiga la curaduría y su relacion con los objetos orientados de la TAR. En esta investigación destaca que los objetos no deben ser estudiados como 'productos', sino como 'procesos' que materializan una práctica social, puestos a prueba por medio de las prácticas curatoriales. Enfatiza aquello que Michael Bandaxall había dicho "no hay exposición sin construcción" (I99I, p. 34). El texto revisa por medio de una etnografía el trabajo de los curadores del Museo de Ciencias de Londres con respecto a una muestra relacionada con la máquina Orámica, que permite 
dibujar el sonido y que fue el principio de la música electrónica. El análisis se realiza desde múltiples puntos de vista del objeto, permitiendo la participación del público por primera vez en ese espacio museístico en la toma de decisiones, entre los participantes destacan los músicos (p. 193-206).

También aquí podría hablar de la investigación doctoral realizada del 2016 al 2018 en el Museo de Arte Alvar y Carmen T. de Carrillo Gil. Durante el desarrollo de la investigación para la exposición "Färg. El racionalismo espontáneo de Waldemar Sjölander", yo realizaba una etnografía para demostrar su devenir.

En un principio el estudio se enfocaba al montaje de una exposición, pero este se convirtió en una etnografía del museo y de la propia producción del arte, ya que actores humanos y no humanos intervinieron y cambiaron su cause, la exposición pactada se pospuso en múltiples ocasiones primero por un sismo, después por el fallecimiento del nieto del fundador del museo y por último al cambiar de presidente en mi país. También se analizó la selección de piezas que estuvieran disponibles, el contacto o no con las galerías, los coleccionistas y los museos que las tuvieran y el espacio que les podría ser asignado museográficamente, ya que algunas esculturas no pudieron formar parte del proyecto por ser demasiado pesadas. Este estudio lo realicé a partir de los pasos que Michel Callon menciona como parte de la traducción ${ }^{1}$ de una red, es decir, la problematización, donde se definen las identidades de los actores; el interesamiento, la seducción u otros mecanismos para definir sus alianzas y su el enrolamiento, la movilización, donde se busca una representación oficial (Galindo, 2019).

El propio Bruno Latour comenzará a hacer experimentos con los objetos en exposiciones. Como mencioné con anterioridad, pare él, el museólogo o el curador se convierte en experimentalista y en estas exposiciones juega con el concepto de heteronomía, definiendo lo exhibido de la siguiente forma:

[...] un conjunto artificial de objetos, instalaciones, personas y argumentos, que no podrían razonablemente ser colocados en cualquier otro lugar. En una exposición las limitaciones habituales del tiempo, el espacio y el realismo se suspenden. Esto significa que se trata de un medio ideal para la experimentación; y especialmente para tratar las

\footnotetext{
I. Mecanismo por medio del cual los mundos social y natural toman forma. El resultado es una situación en la que ciertas entidades controlan a otras. Comprender lo que los sociólogos, por lo general, llaman relaciones de poder, significa describir la manera cómo se define a los actores, cómo se les asocia y simultáneamente se les obliga a permanecer fieles a sus alianzas. El repertorio de la traducción no sólo está concebido para dar una descripción simétrica y tolerante de un proceso complejo que mezcla constantemente una variedad de entidades sociales y naturales. También permite una explicación de cómo unos pocos obtienen el derecho de expresar y representar a los numerosos actores silenciosos de los mundos natural y social que han movilizado. Para analizar este concepto Michel Callon propone los siguientes pasos: problematización, interesamiento, enrolamiento, movilización y disidencia (Callon, 1986, p. 278).
} 
actuales crisis de representación" (Latour, Bruno \& Weibel, Peter, 2005, p. 94).

Este tipo de experimentación de la representación la lleva a cabo en Iconoclash de 2002, explorando la pregunta ¿existe alguna forma de suspender el gesto iconoclasta para interrogarlo en lugar de extenderlo? Donde añade elementos artísticos, religiosos y científicos a partir de la visión de siete curadores. Y la repite en 2005 con Making the things public, cuyo catálogo contiene los textos de 170 autores diferentes en cuyas investigaciones y curadurías se pueden ver las diferentes interpretaciones y representaciones de pueblo, democracia e inclusión, entre otros conceptos.

\section{¿Cómo ve entonces la TAR al museo?}

Como bien lo dice Javier Rodrigo Montero, en el texto que realizó por la conmemoración de los cuarenta años de la Mesa de Santiago en Chile titulado: De las políticas de acceso a las políticas en red. Experiencias de mediación crítica y trabajo en red en museos (2OI2):

El museo no sería un foco centralizador de cultura, ni siquiera un catalizador, sino un mediador ${ }^{1}$ más, dentro de una red de agentes sociales diversos, diferentes e incluso antagonistas [...] se localizaría como un agente social más, un actor en un plano simétrico de relaciones y tensiones con otros agentes, sin demarcar una jerarquía o relación predeterminada.

\section{9}

Y es que al ser el museo un mediador, no se puede catalogar únicamente como un agente social privilegiado (Bourdieu, Pierre \& Darbel, Alain, 2003), ya que puede no serlo, al develar sus complejidades, alterar sus significados y relacionarse con otros agentes sociales.

En este sentido se ubica dentro del espíritu de la Nueva Museología que buscaba ser más cercana a los visitantes, el entorno y el patrimonio (Desvallées, I992) y la Museología Crítica cuyos principios aun nacientes plantean formar

\footnotetext{
I. Para la TAR los actores pueden ser mediadores o intermediarios. Los mediadores producirán asociaciones imprevisibles que vincularán actores de maneras extrañas e irregulares, posiblemente, nada tendrán que ver con la intención original, pues su participación en el flujo de la acción implica alterarla. En cambio los intermediarios, son actores que, dentro de la explicación, llevan significado o fuerza sin transformación. Es decir, todo aquello cuya función sea simplemente la de llevar la declaración -que puede ser una palabra o un objeto - del emisor al siguiente actor, involucrado en la cadena (Latour, I998, p. I09-I42).
} 
ciudadanos más críticos y participativos (Lorente, Jesús Pedro \& Almazán, David, 2003). Al utilizar la TAR, la sociedad no es analizada como objeto sino como el modo de acción asociativa del cual emerge, por ello utiliza al museo convirtiéndolo en un laboratorio de representaciones.

\section{Referencias}

Augé, M. (1996). Los No Lugares. Espacios del anonimato. Barcelona: Gedisa.

Bloor, D. (1976). Knowledge and Social Imagery. Boston: Routledge.

Bloor, D. (1982). Sociologie de la logique ou le limites de l'espistémologie. París: Pandore.

Bandaxall, M. (I99I). Exhibiting Intentions: Some Preconditions of the Visual Display of Culturally Purposeful Objects. En I. \&. Karp, Exhibiting Cultures. The Poetics and Politics of Museum Display (pp. 33-4I). Washington y Londres: Smithsonian Institution Press.

Barnes, B. (1995). Sobre ciencia. Barcelona: RBA Editores.

Bennett, T. (1995). The birth of the museum. History, theory, politics. Londres, Inglaterra: Routledge.

Bourdieu, P., \& Darbel, A. (2003). El amor al arte. Los museos europeos y su público. Barcelona: Paidós Estética.

Callon, M. (1986). Some Elements of a Sociology of Translation: Domestication of the Scallops and the Fishermen of St. Brieuc Bay. En J. Law, Power, Action, and Belief: A New Sociology of Knowledge? Londres, Inglaterra: R.K.P.

Certeau, M. (2007). Relatos del espacio. En M. Certeau, La invención de lo cotidiano (2da. Reimpresión ed., pp. I27-I42). México, México: ITESO/ UIA.

Desvallées, A. (1992). Présentation. En A. Desvallées, Vagues. Une anthologie de la nouvelle muséologie (Vol. I, pp. 2I-39). Lyon, Francia : Diffusion Presses Universitaires de Lyon.

Galindo, S. (2019). La producción social del arte. Redes de cooperación y controversia. México, Mexico: UIA.

Hetherington, K. (1999). From blindness to blindness: museums, heterogeneity and the subject. En J. Law, \& J. Hassard, Actor Network Theory and After (pp. 51-73). Oxford, Inglaterra : Blackwell.

Kang, L. (2006). Object/Shadow-Notes on a Developing Art Form. In H. Becker, R. Faulkner, \& B. Kirshenblatt-Gimblett, Art from Start to Finish (pp. 158-172). Chicago : The University of Chicago Press.

Kjærboe, R. (2016). Collecting the modern. Aarhus, Dinamarca: Aarhus University. 
Latour, B. (1996). On actor-network theory. A few clarifications plus more than a few complications. CSI-Paris/Science Studies-San Diego, 47, 369-38r. Obtenido de Soziale Welt: http://www.cours.fse.ulaval.ca/edc-65804/ latourclarifications. pdf

Latour, B. (1998). La tecnología es la sociedad hecha para que dure. En M. Domenech, \& F. J. Tirado, Sociología simétrica. Ensayos sobre ciencia, tecnología y sociedad (pp. I09-I42). Barcelona, España: Gedisa Editorial.

Latour, B. (I999). DISCUSSION For David Bloor... and Beyond: A Reply to David Bloor's 'Anti-Latour'. Studies in history and philosophy of science, zо(I), ІІз-гзо.

Latour, B. (2008). Reensamblar lo social. Buenos Aires: Manantial.

Latour, B. \& Weibel, P. (2005). Making Things Public. Atmospheres of Democracy. Karlsruhe y Cambridge, Inglaterra: MIT Press and ZKM, Center for Art and Media.

Law, J. \& Mol, A. (1994). Notes on materiality and sociality. The Social Review (43), 274-294.

Lorente, J. P. \& Almazán, D. (2003). Museología crítica y arte contemporáneo. Zaragoza, España: Prensas Universitarias de Zaragoza.

Macdonald, S. (2002). Behind the scenes at the Science Museum. Oxford, Inglaterra : Berg.

Montero, J. R. (20I2). De las políticas de acceso a las políticas en red Experiencias de mediación crítica y trabajo en red en museos. Revista Museos (3I), 74-85.

Núñez, J. J. (20II). La ciencia y la tecnología como procesos sociales. Lo que la educación cientifica no debe olvidar. (O. d. cultura, Productor) Recuperado el or de 06 de 20i6, de Organización de Estados Iberoamericanos para la ciencia y la cultura: http://www.oei.es/salactsi/nunezo5.htm

Ritzer, G. (2005). Encyclopedia of Social Theory. (G. Ritzer, Ed.) Thousand Oaks, California, Estados Unidos de América: SAGE Publications.

Toon, R. (2005). Black box science in black box science centres. In S. MacLeod, Reshaping Museum Space Architecture, Design, Exhibitions (pp. 26-38). Nueva York: Routledge.

Waller, L. (2016). Curating actor-network theory: testing object-oriented sociology in the science museum. Museums\& Society, I4(I), 193-206.

Walsh, K. (1992). The representation of the past. Museums and heritage in the postmodern world. Londres : Routledge. 